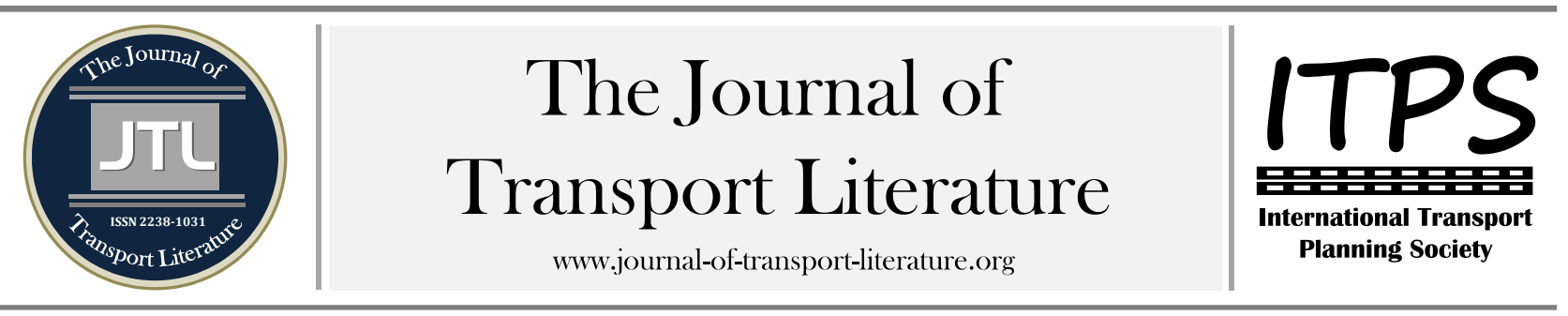

\title{
Avaliação de desempenho do processo logístico de exportação do milho brasileiro: uma aplicação da análise envoltória de dados - DEA
}

Lucas de Oliveira Melo Cicolin; Andréa Leda Ramos de Oliveira ${ }^{+}$

Universidade Estadual de Campinas, UNICAMP, Campinas, Brasil

\section{Article Info}

Palavras-chave:

Exportação Agrícola

Processo Logístico

Análise de Desempenho

Submitted 17 Jun 2015;

received in revised form 5 Jul 2015;

accepted 12 Jul 2015

\section{Licensed under}

Creative Commons

CC-BY 3.0 BR.

\section{Resumo}

Apesar dos avanços significativos da agricultura brasileira, os custos logísticos sobretudo com transporte e armazenagem, continuam sendo as principais barreiras que limitam o potencial do agronegócio brasileiro. Portanto, a avaliação do desempenho do processo logístico brasileiro pode direcionar o desenvolvimento e o investimento necessário ao atendimento da demanda de transportes dos grãos. 0 objetivo do presente estudo foi analisar a eficiência relativa do processo logístico de exportação do milho brasileiro das principais rotas praticadas para o escoamento da produção através do desenvolvimento de um sistema de medição de desempenho que utilizou como metodologia a Análise por Envoltória de Dados (DEA). Para definição dos indicadores de medição de desempenho foi adotado o Balanced Scorecard (BSC) que estruturou o sistema em quatro modelos de análise de eficiência: financeiro, cliente, processos internos do negócio e aprendizado/crescimento. Foi possível definir como rota de referência com melhor desempenho o transporte intermodal rodohidroviário do estado do Mato Grosso até os portos de Santarém - PA e Manaus - AM através do terminal de Miritituba - PA.

+ Corresponding author. Univ. Estadual de Campinas, Faculdade de Engenharia Agrícola. Av. Cândido Rondon, 501. CEP: 13083-875, Campinas/SP. Brasil.

E-mail address: andrea.oliveira@feagri.unicamp.br

\section{Introdução}

0 agronegócio brasileiro destaca-se mundialmente por sua eficiência produtiva. Contudo, a manutenção do sucesso da agricultura brasileira depende de avanços sucessivos em novas tecnologias com ganhos de produtividade e/ou agregação de valor no produto final, entretanto um aspecto recorrente para sustentação do setor agrícola é a superação dos entraves relacionados a infraestrutura logística (Oliveira, 2014).

As limitações do sistema logístico afetam sobremaneira o desempenho da agricultura, desde aspectos de formação de preço até a própria competitividade do setor no cenário internacional. Em especial no caso dos grãos (soja e milho), a interferência é ainda maior, uma vez que contam uma descentralização da produção distante dos portos de exportação e contam com sucessivos aumentos dos volumes exportados, o que faz com que tais produtos demandem um sistema de transporte e armazenagem cada vez mais eficiente (Branco e Caixeta Filho, 2011).

Somente através do direcionamento de investimentos e resolução dos entraves logísticos, como: déficit da capacidade de armazenagem, desburocratização e organização portuária, redistribuição da matriz de transportes de cargas, aumento da capacidade e eficiência ferroviária e hidroviária, possibilitará o aumento da competitividade brasileira (Fleury, 2000).

Além dos entraves financeiros e sociais, a vertente ambiental está se tornando cada vez mais relevante no processo de seleção de fornecedores e parceiros comerciais no cenário mundial, devido ao aumento da preocupação com as práticas limpas e sustentáveis da cadeia de suprimentos, sendo necessária uma análise mais criteriosa e não apenas baseada nos custos (Macedo et al, 2010). Tendo em vista a fragilidade da logística de transporte e armazenagem, qual o nível da eficiência do processo logístico brasileiro de exportação do milho, com relação aos custos econômicos, aos parâmetros ambientais e aspectos operacionais?

Assim, o objetivo geral do estudo foi desenvolver um sistema de medição de desempenho que possibilite a medição da eficiência relativa do processo logístico de exportação do milho através do método da Análise por Envoltória de Dados (DEA) nas principais rotas em volume total exportado. 0 artigo está estruturado em cinco seções, incluindo introdução e conclusões. A primeira conta com a caracterização do processo produtivo-logístico do milho. A seção 2 aborda a metodologia utilizada e a descrição das rotas avaliadas. A análise dos resultados sobre a situação atual do processo logístico do milho brasileiro é desenvolvida na seção 3, e por fim as considerações finais.

\section{Dinâmica do Mercado do Milho}

O complexo agroindustrial do milho é estratégico para a economia nacional, pois além de abastecer outras cadeias produtivas como a avicultura, suinocultura e pecuária na forma de ração, que juntamente com o consumo in natura e destinada à indústria nacional, consumiu 72,2\% dos 79,3 milhões de toneladas produzidos na safra 2013/2014. 0 Brasil se apresenta com um dos principais produtores mundiais de milho, com 8,02\% dos 988,56 milhões de toneladas do total produzido, ficando atrás apenas dos EUA que contribuíram com 353,7 milhões de toneladas (35,78\%) e da China com 218,49 milhões de toneladas (22,10\%) (USDA, 2014). 
Um total de 128,6 milhões de toneladas foram comercializadas no contexto internacional, dos quais 39\% foram produzidos e exportados pelos Estado Unidos, 18\% pelo Brasil (23,4 milhões de toneladas) e 16\% pela Ucrânia USDA (2014).

Segundo dados do MDIC (2014), os estados produtores brasileiros que exportam milho, tem como principal expoente o Mato Grosso (59\% do total exportado), seguido pelo Paraná com (13\%) e Goiás com 12\%. Juntos representam mais de $84 \%$ do volume nacional exportado. Como principais portos brasileiros na exportação do milho estão o porto de Santos - SP (43\%), mais de 10 milhões de toneladas e o porto de Paranaguá - PR, com $15 \%$ do volume total de milho exportado.

Para Oliveira (2014), os constantes desenvolvimentos nos cultivares, impulsionando a produtividade e expandindo a área plantada em regiões antes improdutivas pode fazer aumentar ainda mais a oferta de milho nacional, porém, aspectos como o aumento da eficiência dos portos, certificações internacionais da qualidade do milho produzido, desenvolvimento de malha logística, desburocratização do processo e redução dos custos, ainda são elementos essências para esta cadeia produtiva.

Conforme os dados da CNT (2014), a matriz de transportes brasileira se encontra desequilibrada, com 64\% de toda a carga transportada no país através de rodovias, apenas $22 \%$ pelo modal ferroviário, e $14 \%$ pelo modal hidroviário, uma realidade muito diferente de distribuição entre rodovia, ferrovia e hidrovia em países como Estados Unidos $(32 \%$, 43\%, $25 \%)$ e Rússia $(8 \%, 81 \%, 11 \%)$. A distribuição ideal no transporte de grãos, como é o caso do milho, seria por ferrovias e hidrovias, com a atuação do modal rodoviário direcionado ao abastecimento de terminais intermodais próximos aos centros produtores (Morales et al, 2013).

As dificuldades na infraestrutura brasileiro não se limitam aos transportes, mas também a rede armazenagem. Além do déficit de capacidade, a localização é inadequada concentrada nos centros urbanos, o ideal seria que as unidades se concentrassem nas propriedades rurais e na zona rural. Com argumentam Junqueira e Morabito (2012), o armazenamento é um fator estratégico no setor agrícola, pela capacidade de recebimento e conservação da produção para posterior distribuição equalizada da produção corrigindo o fator sazonalidade, auxiliando na sustentação da demanda durante todo o ano, além de manter a estabilidade dos preços e dos fretes.

\section{Metodologia}

No processo de estruturação do sistema de análise de eficiência, foram revisados os principais sistemas de medição de desempenho. O Balanced Scorecard foi desenvolvido por Kaplan e Norton, publicado 1992 na Harward Bussiness Review, o principal objetivo da metodologia é traduzir a estratégia da empresa em indicadores de desempenho, desdobrando-a em quatro perspectivas: financeira; do cliente; dos processos internos da empresa; e do aprendizado e crescimento, não restringindo a análise à critérios financeiros (Kaplan e Norton, 1997).

A metodologia Data Envelopment Analysis (DEA, em português análise por envoltória de dados), que teve origem com Farrel (1957) e generalizada por Charnes, Cooper e Rhodes em (1978), caracteriza-se como uma técnica não paramétrica, que permite lidar com várias saídas (outputs) e entradas (inputs) para medir, comparativamente, o desemprenho de unidades independentes, ou seja, à eficiência de cada unidade. A técnica DEA acontece por meio de uma programação linear padrão, que busca estabelecer a eficiência máxima de uma DMU, expressa na razão entre inputs e outputs, comparando a performance de uma unidade em relação ao grupo de unidades semelhantes. A aplicação da técnica nos permite dizer quais unidades tem um desempenho mais eficiente (Azambuja et al., 2015).

Conforme Azambuja et al. (2015), os modelos DEA podem trabalhar com retornos de escala constantes (modelo CCR Charles, Cooper e Rhodes, também conhecido como CRS) ou com retornos de escala variáveis (modelo BCC - Banker, Charnes e Cooper, também conhecido como VRS). No presente estudo foi utilizado o modelo BCC orientado a output que tem sua formulação matemática definida, segundo Soares de Mello et al (2005):

Sujeito a:

$$
\operatorname{Max} h_{o}
$$

$$
\begin{gathered}
x_{j o}-\sum_{k=1}^{n} x_{i k} \cdot \lambda_{k} \geq 0, \forall i \\
-h_{o} \cdot y_{j o}+\sum_{\mathrm{k}=1}^{\mathrm{n}} y_{j k} \cdot \lambda_{k} \geq 0, \forall j \\
\sum_{\mathrm{k}=1}^{\mathrm{n}} \lambda_{k}=1
\end{gathered}
$$

0 modelo proposto maximiza as saídas mantendo inalteradas as entradas. Em (1), $h_{o}$ é a eficiência e $\lambda_{k}$ é a contribuição da DMU $k$ na formação do alvo da DMU $o$. No desenvolvimento do estudo foi realizado o mapeamento do processo logístico do milho, e as principais rotas de escoamento para exportação, que foram definidos como objetos de estudo, chamadas de DMU's. Foram então definidas as rotas que são objeto de estudo. Foram definidas 17 rotas, desta 8 são unimodal utilizando apenas o modal rodoviária e 9 são intermodais utilizando a combinação dos modais rodoviário/ferroviário e rodoviário/hidroviário. Assim, temos origens das rotas nos estados do Mato Grosso, Paraná e Goiás e destinos aos Portos de Santos (SP), Paranaguá (PR), São Francisco do Sul (SC), Vitória (ES), Santarém (PA) e Manaus (AM). Por exemplo a rota MTSP-RODO (DMU01) é caracterizada pelo fluxo de milho entre o estado produtor de Mato Grosso ao porto de Santos (SP) utilizando a opção de transporte exclusiva rodoviária. Já a MT-SP-RODO-FERRO (DMU02) considera a rota entre o estado produtor de Mato Grosso ao porto de Santos (SP) utilizando a opção de transporte intermodal rodoferroviário. 0 milho percorre aproximadamente $600 \mathrm{~km}$ utilizando as rodovias BR-163 e BR-364 até o terminal intermodal de RondonópolisMT, percorrendo o restante do percurso via ferrovia por $1.551 \mathrm{~km}$. Os terminais de transbordo ferroviário considerados 
foram Rondonópolis-MT, Araguari-MG, Maringá-PR e Roncador-GO). Quanto aos terminais hidroviários foram considerados o Terminal de Miritituba-PA (DMU06) e o Terminal de Porto Velho-RO (DMU07).

\subsection{Definição das Variáveis}

Para estruturação das variáveis foi utilizado como referência o Balanced Scorecard (BSC) desdobrando a estratégia "competitividade do processo logístico através de práticas sustentáveis" de acordo com as dimensões Financeira, dos Processos Internos de Negócios, do Cliente e de Aprendizado e Crescimento (Quadro 1).

Dimensão Financeira: Esta dimensão representa os custos e analisa se todos os indicadores estão contribuindo de modo a minimizar o custo final do milho brasileiro no mercado internacional. 0 objetivo estratégico que representa esta dimensão é o Custo Logístico, custo total da entrega do milho brasileiro no porto de Xangai-China, por ser o maior porto em atividade no mundo, e pelo mercado consumidor do grão brasileiro se concentrar na Ásia.

Dimensão do Cliente: Na perspectiva do cliente, foi estabelecido o indicador de Participação da DMU, que representa o total relativo exportado pela rota utilizada (DMU) em relação ao total de milho brasileiro exportado.

Dimensão dos Processos Internos de Negócios: Nesta dimensão são avaliadas as atividades que devem ser desenvolvidas, levando em conta histórico de processos burocráticos de aduana e excessivo tempo para entrega. 0 objetivo estratégico que analisa esses fatores é o Lead Time de Entrega, considerando a fila de espera dos navios no porto e o tempo de transporte até o porto de Xangai-China.

Dimensão de Aprendizado e Crescimento: Na perspectiva de aprendizado e crescimento, são analisados os fatores que devem ser desenvolvidos para o sucesso futuro dos negócios. 0 objetivo adotado representa a adoção de práticas limpas, adotou-se o Desenvolvimento Sustentável que mede o impacto do escoamento da produção de milho de acordo com os modais e a rota utilizados. A estimativa adotada neste caso foi a emissão de $\boldsymbol{C O}_{2}$.

Após a análise dos indicadores propostos na literatura, foram selecionados com indicadores de input aqueles que traduzem a visão estratégia da cadeia de suprimentos utilizados no estudo (Quadro 1). Com o objetivo de verificar a relação de cada indicador de input nos indicadores estratégicos - output, foi estruturado o Quadro 1, que apresenta os quatro modelos e os respectivos indicadores que compõe a análise.

\begin{tabular}{ll} 
Quadro 1 - Modelos de medição de eficiência. Fonte: Elaborado pelo autores. \\
\hline Output & Input \\
\hline Custo Logístico & Composição da matriz de transportes \\
(Dimensão Financeira) & Disponibilidade de armazéns \\
& Custo de produção do milho \\
& Produtividade do milho no campo \\
\hline Participação da DMU & Custo total \\
(Dimensão do Cliente) & Fila nos portos/navios \\
\hline \multirow{2}{*}{ Lead time de entrega } & Extensão da rota \\
(Dimensão dos Processos Internos de Negócios) & Velocidade média \\
\hline \multirow{2}{*}{ Desenvolvimento Sustentável } & Fila nos portos/navios \\
(Dimensão de Aprendizado e Crescimento) & Extensão da rota \\
\hline
\end{tabular}

No processo de resolução dos modelos utilizou-se o método de retorno variável de escala (BCC). Como forma de tratar os outputs indesejáveis, os dados foram tratados de forma a ajusta-los através do método da abordagem multiplicativa inversa, que transforma os indicadores de output indesejáveis (Custo Logístico, Lead Time de Entrega e Desenvolvimento Sustentável) como inputs através da fórmula $F_{(u)}=-u$. Com o objetivo de aumentar a discriminação entre as DMU's eficientes, foi utilizado o método da Fronteira Invertida, que após o cálculo da eficiência relativa com os dados do modelo, é calculada a ineficiência do modelo, para tal é necessário inverter os inputs pelos outputs, após os cálculos da eficiência e ineficiência, é calculada a média entre os resultados.

$$
\text { Eficiência da Fronteira Invertida }=\frac{\text { Eficiência Padrão }+(1-\text { Ineficiência })}{2}
$$

\section{Resultados e discussão}

Foram analisados inicialmente os resultados obtidos pelas rotas em cada um dos quatro modelos propostos: custo logístico/dimensão financeira, participação da DMU/dimensão cliente, lead time de entrega/dimensão processos internos e desenvolvimento sustentável/dimensão crescimento. Num segundo momento, nas conclusões, as rotas foram analisadas com um todo.

\subsection{Análise dos resultados: dimensão financeira}

Após a resolução do modelo financeiro, foi verificado como referência a rota intermodal rodohidroviária através do terminal de Miritituba-PA por ter obtido um índice de eficiência geral de 76\%, sendo a mais eficiente entre as rotas estudadas. A partir desta rota foi então calculada a eficiência relativa das outras rotas, com destaque para a rota intermodal rodoferroviária de exportação do milho do estado de Goiás ao porto de Santos-SP através do terminal de Roncador-GO utilizando as ferrovias FCA e ALL Malha Paulista obteve um índice de eficiência relativa de 90,7\%, a Tabela 1 apresenta os resultados do modelo financeiro.

\subsection{Análise dos resultados: dimensão cliente}

Como principais referências no modelo de participação das rotas foi verificado o processo logístico de escoamento do milho entre o estado produtor do Mato Grosso e o porto de Santos - SP via rodoviária que obteve um índice de $98 \%$ de eficiência, resultado que já era esperado por se tratar do maior estado exportador e do maior porto brasileiro em volume de exportação, com a distribuição de $74 \%$ do total transportado de acordo com a matriz de transportes brasileira da CNT (2014). Este resultado demonstra que a rota se apresenta no limite de sua capacidade, havendo a necessidade de distribuição por outras rotas, sendo a principal alternativa a utilização dos portos de Santarém - PA e de Manaus - AM, com índice de eficiência relativa de $96,3 \%$, seguidos das alternativas utilizando a rodovia para transporte entre o estado do Paraná e os portos de Paranaguá - PR e São Francisco do Sul - SC com índices de eficiência relativa de 94,4\% e 87,9\%, respectivamente, conforme detalhamento dos resultados disponível na Tabela 2. 
Tabela 1 - Eficiência Final das DMU's, modelo financerio/custo logísticos. Fonte: Dados da pesquisa (2015).

\begin{tabular}{llcccl}
\hline DMU / Indicador & DMU & Eficiência & Ineficiência & $\begin{array}{l}\text { Eficiência da Fronteira } \\
\text { Invertida }\end{array}$ & $\begin{array}{l}\text { Eficiência } \\
\text { Final }\end{array}$ \\
\hline MT-SP-RODO & & & & 0,366957 & 0,482665 \\
MT-SP-RODO-FERRO & DMU01 & 0,572050 & 0,838137 & 0,313002 & 0,806293 \\
MT-PR-RODO & DMU02 & 1,000000 & 0,773996 & 0,61302 & 0,409616 \\
MT-PR-RODO-FERRO & DMU03 & 0,529067 & 0,906229 & 0,311419 & 0,386762 \\
MT-PA/AM-RODO & DMU04 & 0,532161 & 0,944073 & 0,294044 & 0,811756 \\
MT-PA/AM-RODO-HIDRO & DMU05 & 0,819424 & 0,585113 & 0,617155 & 1,000000 \\
MT-PA/AM-RODO-HIDRO 2 & DMU06 & 1,000000 & 0,479456 & 0,760272 & 0,660348 \\
MT-ES-RODO & DMU07 & 0,767949 & 0,763861 & 0,502044 & 0,315319 \\
MT-ES-RODO-FERRO & DMU08 & 0,479456 & 1,000000 & 0,239728 & 0,63708 \\
PR-PR - RODO & DMU09 & 0,637890 & 0,993624 & 0,322133 & 0,789866 \\
PR-PR - RODO-FERRO & DMU10 & 0,880385 & 0,907441 & 0,486472 & 0,525402 \\
PR-SC-RODO & DMU11 & 1,000000 & 0,798897 & 0,600551 & 0,656878 \\
PR-SC- RODO-FERRO & DMU12 & 0,798897 & 1,000000 & 0,399449 & 0,752041 \\
GO-SP-RODO & DMU13 & 0,893217 & 0,894404 & 0,499406 & 0,907856 \\
GO-SP-RODO-FERRO & DMU14 & 0,891240 & 0,747728 & 0,571756 & 0,438267 \\
GO-ES-RODO & DMU15 & 1,000000 & 0,619565 & 0,690217 & 0,657659 \\
GO-ES-RODO-FERRO & DMU16 & 0,666405 & 1,000000 & 0,333202 & \\
\hline
\end{tabular}

Tabela 2 - Eficiência das DMU's, modelo cliente/participação da DMU. Fonte: Dados da pesquisa (2015).

\begin{tabular}{|c|c|c|c|c|c|}
\hline DMU / Indicador & DMU & Eficiência & Ineficiência & $\begin{array}{l}\text { Eficiência da Fronteira } \\
\text { Invertida }\end{array}$ & $\begin{array}{l}\text { Eficiência } \\
\text { Final }\end{array}$ \\
\hline MT-SP-RODO & DMU01 & 1,00 & 0,03861 & 0,98070 & 1,00000 \\
\hline MT-SP-RODO-FERRO & DMU02 & 0,38 & 0,11232 & 0,63270 & 0,64515 \\
\hline MT-PR-RODO & DMU03 & 0,11 & 0,34375 & 0,38539 & 0,39297 \\
\hline MT-PR-RODO-FERRO & DMU04 & 0,04 & 1,00000 & 0,01968 & 0,02007 \\
\hline MT-PA/AM-RODO & DMU05 & 1,00 & 0,10938 & 0,94531 & 0,96392 \\
\hline MT-PA/AM-RODO-HIDRO & DMU06 & 1,00 & 1,00000 & 0,50000 & 0,50984 \\
\hline MT-PA/AM-RODO-HIDRO 2 & DMU07 & 0,11 & 1,00000 & 0,05469 & 0,05576 \\
\hline MT-ES-RODO & DMU08 & 0,27 & 1,00000 & 0,13569 & 0,13836 \\
\hline MT-ES-RODO-FERRO & DMU09 & 0,09 & 1,00000 & 0,04664 & 0,04756 \\
\hline PR - PR - RODO & DMU10 & 1,00 & 0,14796 & 0,92602 & 0,94425 \\
\hline PR - PR - RODO-FERRO & DMU11 & 1,00 & 0,43044 & 0,78478 & 0,80023 \\
\hline PR - SC - RODO & DMU12 & 1,00 & 0,27538 & 0,86231 & 0,87928 \\
\hline PR - SC - RODO-FERRO & DMU13 & 1,00 & 0,80111 & 0,59945 & 0,61125 \\
\hline GO-SP-RODO & DMU14 & 0,48 & 0,18122 & 0,64713 & 0,65987 \\
\hline GO-SP-RODO-FERRO & DMU15 & 0,19 & 0,52719 & 0,33268 & 0,33923 \\
\hline GO-ES-RODO & DMU16 & 0,19 & 0,33036 & 0,43078 & 0,43926 \\
\hline GO-ES-RODO-FERRO & DMU17 & 0,06 & 0,96105 & 0,04899 & 0,04996 \\
\hline
\end{tabular}

\subsection{Análise dos resultados: dimensão processos internos}

Como resultado do modelo de eficiência de processos internos de negócios, todas as rotas apresentaram índice de eficiência parcial acima de 93\%, devido à representatividade do tempo de transbordo marítimo entre os portos brasileiros e o porto de Xangai - China, que influencia diretamente no Lead Time total do processo. Após a aplicação do método da fronteira invertida, as rotas analisadas tiveram um índice de ineficiência muito alto (acima dos 90\%), sendo assim a eficiência da fronteira invertida apresentou índices abaixo de 60\%. As rotas destaques deste modelo foram do estado do Mato Grosso para os portos de Santarém-PA / Manaus-AM utilizando a rota intermodal rodohidroviária através do terminal de Miritituba - PA, e a rota Paraná - Porto de São Francisco do Sul - SC que utiliza a ferrovia NOVOESTE apresenta um índice de eficiência relativa de $98 \%$. Os dados relativos ao modelo de processos internos de negócios estão disponíveis na Tabela 3.

Tabela 3 - Eficiência das DMU's, modelo processos internos / lead time de entrega. Fonte: Dados da pesquisa (2015).

\begin{tabular}{|c|c|c|c|c|c|}
\hline DMU / Indicador & DMU & Eficiência & Ineficiência & $\begin{array}{l}\text { Eficiência da Fronteira } \\
\text { Invertida }\end{array}$ & $\begin{array}{l}\text { Eficiência } \\
\text { Final }\end{array}$ \\
\hline MT-SP-RODO & DMU01 & 0,94795 & 0,98711 & 0,48042 & 0,86976 \\
\hline MT-SP-RODO-FERRO & DMU02 & 0,94247 & 0,98342 & 0,47953 & 0,86814 \\
\hline MT-PR-RODO & DMU03 & 0,93573 & 1,00000 & 0,46787 & 0,84703 \\
\hline MT-PR-RODO-FERRO & DMU04 & 0,93260 & 1,00000 & 0,46630 & 0,84419 \\
\hline MT-PA/AM-RODO & DMU05 & 1,00000 & 0,93573 & 0,53214 & 0,96338 \\
\hline MT-PA/AM-RODO-HIDRO & DMU06 & 1,00000 & 0,89527 & 0,55236 & 1,00000 \\
\hline MT-PA/AM-RODO-HIDRO 2 & DMU07 & 1,00000 & 0,93869 & 0,53065 & 0,96070 \\
\hline MT-ES-RODO & DMU08 & 0,95657 & 1,00000 & 0,47829 & 0,86589 \\
\hline MT-ES-RODO-FERRO & DMU09 & 0,91415 & 1,00000 & 0,45707 & 0,82749 \\
\hline PR - PR - RODO & DMU10 & 1,00000 & 0,96480 & 0,51760 & 0,93706 \\
\hline PR - PR - RODO-FERRO & DMU11 & 1,00000 & 0,96458 & 0,51771 & 0,93726 \\
\hline PR - SC - RODO & DMU12 & 1,00000 & 0,95489 & 0,52256 & 0,94604 \\
\hline PR - SC - RODO-FERRO & DMU13 & 1,00000 & 0,91000 & 0,54500 & 0,98667 \\
\hline GO-SP-RODO & DMU14 & 0,97808 & 0,96604 & 0,50602 & 0,91610 \\
\hline GO-SP-RODO-FERRO & DMU15 & 1,00000 & 1,00000 & 0,50000 & 0,90520 \\
\hline GO-ES-RODO & DMU16 & 0,97956 & 0,95525 & 0,51216 & 0,92721 \\
\hline GO-ES-RODO-FERRO & DMU17 & 1,00000 & 0,97987 & 0,51006 & 0,92342 \\
\hline
\end{tabular}

Tabela 4 - Eficiência das DMU's, modelo crescimento / desenvolvimento sustentável. Fonte: Dados da pesquisa (2015).

\begin{tabular}{llllll}
\hline DMU / Indicador & DMU & Eficiência & Ineficiência & $\begin{array}{l}\text { Eficiência da Fronteira } \\
\text { Invertida }\end{array}$ & $\begin{array}{l}\text { Eficiência } \\
\text { Final }\end{array}$ \\
\hline MT-SP-RODO & & & & 0,12882 & 0,13204 \\
MT-S-RODO-FERRO & DMU01 & 0,06084 & 0,80321 & 0,31880 & 0,32678 \\
MT-PR-RODO & DMU02 & 0,13159 & 0,49400 & 0,08696 & 0,08913 \\
MT-PR-RODO-FERRO & DMU03 & 0,05544 & 0,88153 & 0,13269 \\
MT-PA/AM-RODO & DMU04 & 0,06696 & 0,80806 & 0,12945 & 0,29013 \\
MT-PA/AM-RODO-HIDRO & DMU05 & 0,11830 & 0,55221 & 0,28304 & 0,33702 \\
MT-PA/AM-RODO-HIDRO 2 & DMU06 & 0,13664 & 0,47907 & 0,32878 & 0,14072 \\
MT-ES-RODO & DMU07 & 0,06317 & 0,78861 & 0,13728 & 0,02505 \\
MT-ES-RODO-FERRO & DMU08 & 0,04887 & 1,00000 & 0,02444 & 0,03559 \\
PR - PR - RODO & DMU09 & 0,06944 & 1,00000 & 0,03472 & 0,91760 \\
PR - PR - RODO-FERRO & DMU10 & 1,00000 & 0,20964 & 0,89518 & 1,00000 \\
PR - SC- RODO & DMU11 & 1,00000 & 0,04887 & 0,97557 & 0,76489 \\
PR-SC- RODO-FERRO & DMU12 & 0,73216 & 0,23976 & 0,74620 & 0,89426 \\
GO-SP-RODO & DMU13 & 0,80549 & 0,06067 & 0,87241 & 0,42624 \\
GO-SP-RODO-FERRO & DMU14 & 0,23325 & 0,40161 & 0,41582 & 0,54037 \\
GO-ES-RODO & DMU15 & 0,24990 & 0,19556 & 0,52717 & 0,28273 \\
GO-ES-RODO-FERRO & DMU16 & 0,11389 & 0,56225 & 0,27582 & 0,46854 \\
\hline
\end{tabular}




\subsection{Análise dos resultados: dimensão crescimento}

As rotas que obtiveram destaque têm como origem o estado do Paraná, que é o estado produtor mais próximo das vias de exportação. A referência é a rota ferroviária via até o porto de Paranaguá - PR com eficiência final de 97,5\%, seguido da rota rodoviária pela BR - 376 até o porto de Paranaguá - PR (91,7\% de eficiência relativa), e das rotas até o porto de São Francisco do Sul - SC, $(89,4 \%$ de eficiência relativa). O detalhamento dos resultados de todas as rotas é apresentado na Tabela 4.

\section{Conclusões}

A presente pesquisa analisou a eficiência das principais rotas de escoamento da produção de milho brasileiro para exportação entre os principais estados produtores e os principais portos brasileiros. No processo de avaliação foram definidos os indicadores de entrada e saída que representavam influência no resultado final do processo.

Através da análise dos quatro modelos propostos: financeiro, participação das rotas, de processos internos de negócios e de desenvolvimento sustentável, pode-se destacar a rota de exportação do milho DMU06 que representa o fluxo do Estado do Mato Grosso para os portos de Santarém-PA / Manaus-AM, utilizando a rota intermodal rodohidroviária através do terminal hidroviário de Miritituba-PA na Hidrovia Tapajós-Amazonas (MT-PA/AM-RODO-HIDRO - DMU06), que foi

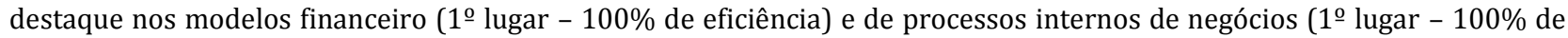
eficiência), no modelo de desenvolvimento sustentável se apresentou como melhor alternativa quando a origem do milho é o Estado do Mato Grosso - maior estado produtor. Quando a referência é o Estado do Paraná, pode-se destacar a rota rodoferroviária até o porto de Paranaguá - PR, e quando o milho tem origem no estado de Goiás, a rota intermodal rodoferroviária pelo porto de Santos - SP é a referência.

Através do modelo de avaliação de eficiência financeira, foi possível verificar a influência da participação dos modais ferroviário e hidroviário no processo de definição de rotas eficientes, tal qual 4 dos 5 melhores resultados contavam com a intermodalidade. É importante ressaltar também que o escoamento para os portos de Santarém - PA e Manaus - AM se apresentam como melhores alternativas ao já sobrecarregado porto de Santos - SP. Já no modelo de análise de eficiência em desenvolvimento sustentável, as rotas que apresentavam uma extensão menor entre o estado produtor e o porto de exportação foram privilegiadas, como foi o caso da rota do Estado do Paraná até o porto de Paranaguá - PR através da intermodalidade rodoferroviária. Os resultados desse estudo permitem a análise do nível de desenvolvimento de cada rota e o direcionamento de investimentos nas rotas que apresentaram resultados insatisfatórios, ou seja, baixos índices de eficiência. Os modelos propostos se tornam relevantes devido a sua possibilidade de replicação na análise de eficiência de outros processos logísticos para outras commodities agrícolas, bem como na comparação com os países concorrentes na cadeia do milho.

\section{Referências}

Azambuja, A. M. V., M. S. Oliveira, \& M. L. P. Lima. (2015). Aplicação do Modelo de Análise de Janelas DEA em Terminais de Contêineres Brasileiros. Journal of Transport Literature, 9 (4), 25-29.

Branco, J. E. H., \& Caixeta Filho, J. V. (2011) Estimativa da demanda de carga captável pela estrada de ferro Norte-Sul. Journal of Transport Literature, 5 (4), 17-50.

Charnes, A., W. W. Cooper, \& E. Rhodes. (1978). Measuring the efficiency of decision-making units. European Journal of Operational Research, 2, 429-444.

CNT. (2014). Confederação Nacional dos Transportes. Boletim Estatístico.

Fleury, P. F. (2000). A logística brasileira em perspectiva. In: P. F. Fleury, P. F. Wanke, \& K. F. Figueiredo (Orgs.). Logística empresarial: a perspectiva brasileira (pp. 15-22). São Paulo: Atlas.

Junqueira, R. A. R., \& Morabito, R. (2012). Production and logistics planning considering circulation taxes in a multi-plant seed corn company, Computers and Electronics in Agriculture, 84, 100-110.

Kaplan, R. S., Norton, D. P. (1997). A estratégia em ação: balanced scorecard. Rio de. Janeiro: Campus.

Macedo, M. A., J. C. C, B S. Mello, \& E. G. Gomes. (2010). Modelo para redistribuição de cotas de emissão de gases do efeito estufa baseado em medidas de eficiência técnica. Gestão da Produção, 17 (3), 513-524.

MDIC. (2014). Ministério do Desenvolvimento, Indústria e Comércio Exterior. Estatísticas do Comércio Exterior - Sistema Aliceweb.

Oliveira, A. L. R. (2014). A logística do agronegócio: para além do apagão logístico. In: A. M. Buainain, E. Alves, J. M. F. J. Silveira, \& Z. Navarro (Orgs.). 0 mundo rural no Brasil do século 21: a formação de um novo padrão agrário e agrícola (pp. 337-370). Brasília: Embrapa.

Soares de Mello, J. C. C. B, et al. (2005). Curso de Análise de Envoltória de Dados, Anais do XXXVII Simpósio Brasileiro de Pesquisa Operacional (SBPO), Gramado, Brasil.

USDA. (2014). United States Department of Agriculture. Commodities and Products.

\section{Abstract}

Despite significant advances in Brazilian agriculture, logistics costs especially with transport and storage, remain the main barriers that limit the potential of Brazilian agribusiness. Therefore, the evaluation of the Brazilian logistics process performance can drive development and the investment required to meet the grain transport demand. The aim of this study was to analyze the relative efficiency of the logistics of the Brazilian corn export process of the main routes in force for the flow of production through the development of a performance measurement system that used as the methodology for data envelopment analysis (DEA). To define the performance measurement indicators was adopted the Balanced Scorecard (BSC), which structured the system on four models of efficiency analysis: financial, customer, internal business processes and learning / growth. The results shows that the reference route with better performance was the intermodal transport (road and waterway) from Mato Grosso state to the ports of Santarém - PA and Manaus - AM through the waterway terminal Miritituba - PA.

Key words: Agriculture exports, Logistical process, Performance analysis. 